\title{
Lower gingival squamous cell carcinoma with brain metastasis during long-term cetuximab treatment: A case report
}

\author{
TOMOFUMI NARUSE, MITSUKO TOKUHISA, SOUICHI YANAMOTO, YUKI SAKAMOTO, \\ KOHEI OKUYAMA, HIROKI TSUCHIHASHI and MASAHIRO UMEDA \\ Department of Clinical Oral Oncology, Graduate School of Biomedical Sciences, \\ Nagasaki University, Nagasaki 852-8588, Japan
}

Received September 12, 2017; Accepted February 16, 2018

DOI: $10.3892 / \mathrm{ol} .2018 .8261$

\begin{abstract}
Long-term cetuximab treatment can lead to acquired resistance, and tumor progression and/or new lesions often occur. The present report describes a case of lower gingival squamous cell carcinoma with brain metastasis during long-term cetuximab treatment in a 60-year-old man, including findings of an immunohistochemical study. The resected primary tumors, biopsy of the lung metastasis before administration of cetuximab, and brain metastasis specimens mediated by cetuximab were immunohistochemically examined. Histologically, the metastatic brain lesion showed hyperkeratinizing tumor cells with deeply stained irregular nuclei with necrotizing tumor cells, and a decrease in cell density was exhibited in part of the tumor nest. Moreover, the brain lesion was less malignant compared with the primary tumor and metastatic lung lesions. Immunohistochemically, the metastatic brain lesions showed low expression of epidermal growth factor receptor (EGFR) and high expression of $\mathrm{N}$-cadherin compared with the primary tumor and metastatic lung lesions. These results suggest that acquired resistance to cetuximab may be associated with low EGFR expression and increased epithelial-to-mesenchymal transition potential.
\end{abstract}

Correspondence to: Dr Tomofumi Naruse, Department of Clinical Oral Oncology, Graduate School of Biomedical Sciences, Nagasaki University, 1-7-1 Sakamoto, Nagasaki 852-8588, Japan

E-mail: naruse12@nagasaki-u.ac.jp

Abbreviations: EGFR, epidermal growth factor receptor; NCCN, National Comprehensive Cancer Network; R/M, recurrent/metastatic; KRAS, Kirsten rat sarcoma viral oncogene homolog; CT, computed tomography; HNSCC, head and neck squamous cell carcinoma; CRT, chemoradiotherapy; DM, distant metastasis; MRI, magnetic resonance imaging; BBB, blood brain barrier; BTB, blood-tumor barrier; EMT, epithelial-to-mesenchymal transition

Key words: oral squamous cell carcinoma, brain metastasis, cetuximab resistance, epidermal growth factor receptor, epithelial-to-mesenchymal transition

\section{Introduction}

The National Comprehensive Cancer Network (NCCN) Clinical Practice Guideline has suggested that platinum-based systemic therapy including cetuximab, an epidermal growth factor receptor (EGFR) inhibitor, is the gold standard treatment and leads to an increase in progression-free survival and overall survival in recurrent/metastatic $(\mathrm{R} / \mathrm{M})$ head and neck squamous cell carcinoma (HNSCC) $(1,2)$. Other studies have suggested that the addition of cetuximab to weekly paclitaxel is effective for patients who present resistance or are not candidates for platinum-based chemotherapy (3). In contrast, long-term administration can lead to acquired resistance to cetuximab in tumor cells, and tumor progression or metastasis are often experienced. Although several molecular mechanisms, such as Kirsten rat sarcoma viral oncogene homolog (KRAS) mutations and the constitutive activation of EGFR and its downstream pathways, have been reported to be involved in cetuximab resistance (4), histopathological and immunohistochemical changes mediated by cetuximab have not yet been investigated, and the details underlying the mechanisms of resistance remain unclear.

We describe our experience with a case of lower gingival squamous cell carcinoma with isolated brain metastasis during long-term cetuximab treatment, including findings of an immunohistochemical study.

\section{Case report}

A 60-year-old man with a history of lumbar compression fracture was referred to the Nagasaki University Hospital with a complaint of pain in the left mandibular gingiva. Consent was provided to publish the present data. He had smoked 30 cigarettes per day for 40 years and also reported an alcohol intake of two glasses of shochu water per day for 30 years. Intraoral examination revealed a $35 \times 30 \mathrm{~mm}$ elastic hard mass with a central ulceration involving the left mandibular gingiva, which was immediately evaluated via imaging and incisional biopsy. Contrast-enhanced axial computed tomography (CT) showed a $32 \times 23 \mathrm{~mm}$ poorly marginated lesion with bone destruction that extended along the buccal and lingual surfaces of the mandible, but no regional lymph node involvement (Fig. 1). The biopsy specimen exhibited signs of well-differentiated 
squamous cell carcinoma (SCC) invading the submucosal tissue from the mucosal epithelium, and he was diagnosed with SCC of the mandible (T4N0M0 stage IVA). Under general anesthesia, he underwent segmental mandibulotomy, modified radical neck dissection, and reconstruction with fibula osteocutaneous free flaps. However, because unresectable regional recurrence occurred between the splenius muscle and levator scapulae muscle 2 months after surgery, he underwent high dose platinum-based chemoradiotherapy (CRT) for curative treatment (Fig. 2). Radiotherapy (RT) was performed using a total of $66 \mathrm{~Gy}$. Concomitant RT was administered at $2 \mathrm{~Gy} / \mathrm{d}$ for $5 \mathrm{~d} / \mathrm{wk}$. Chemotherapy consisted of a total of $200 \mathrm{mg}$ cisplatin per square meter of body surface during a course of RT. However, multiple lesions occurred in the lung fields during the CRT. Transbronchial biopsy was performed by the department of respiratory medicine, and metastasis of oral squamous cell carcinoma (OSCC) was pathologically diagnosed (Fig. 3). Because he experienced failure of a platinum-based therapy, cetuximab plus paclitaxel were started. Cetuximab was administered at a dose of $400 \mathrm{mg}$ per square meter of body for the first injection and $250 \mathrm{mg}$ per square meter of body weekly thereafter. Paclitaxel was administered weekly at a dose of $80 \mathrm{mg}$ per square meter of body. After 57 courses were administered, as speech disturbances and decreased cognitive function were apparent, brain enhanced magnetic resonance imaging (MRI) was performed and brain distant metastases (DMs) were detected, in spite of better loco-regional and lung DM control. Palliative surgery against the brain metastases was performed by the department of neurosurgery, and metastasis of OSCC was pathologically diagnosed (Fig. 4). Following surgery, the best supportive care has been provided by another hospital and he subsequently died because of cachexia 2 months after palliative surgery.

Pathological findings revealed that primary tumors showed keratinizing tumor cells that had relatively round or cord-like tumor nests with deeply stained irregular nuclei, and tumor cells infiltrated from the gingival epithelium to the submucosa, surrounding the muscle layer and bone marrow of the mandible. Lung DM lesions showed keratinizing tumor cells that had round tumor nests with irregular nuclei and prominent nucleoli. In contrast, brain DM lesions showed a neoplastic appearance comprising hyperkeratinizing tumor cells with deeply stained irregular nuclei, but necrotizing tumor cells and a decrease in cell density were also seen in part of tumor nest. Taken together, tumor cellularity was lower compared to the primary tumor and lung DM lesion due to the administration of cetuximab (Fig. 5).

Immunohistochemistry was performed using paraffin-embedded sections of resected primary tumors, biopsy lung DM specimens, and brain DM specimens. The following primary antibodies were used: EGFR (D38B1; dilution 1:50; CST, Tokyo, Japan), EGFRv3 (9E05M52; dilution 1:200; Bioss Inc., Woburn, MA, USA), CD44v6 (ab78960; dilution 1:100; Abcam, Cambridge, UK), ABCG2 (ab3380; dilution 1:50; Abcam), E-cadherin (M3612; dilution 1:50; Dako; Agilent Technologies, Inc., Glostrup, Denmark), and N-cadherin (M3618; dilution 1:50; Dako; Agilent Technologies, Inc.). Immunohistochemistry revealed that EGFR was strongly expressed primarily in the cell membrane of tumor cells in the primary tumor and lung DM lesions. Strong expression was especially observed at the invasive front. By contrast, in the brain DM lesions, EGFR was weakly or not expressed in the tumor nests, and a marked difference between the lung and brain lesions was observed. EGFRv3 was weakly or not expressed in the primary tumor, lung DM specimens, and brain DM specimens. CD44v6 was strongly expressed in the primary tumor, lung DM specimens, and brain DM specimens, and no marked difference in these markers was observed between the lung and brain DM (Fig. 6A). ABCG2 was weakly or not expressed in the primary tumor, lung DM specimens, and brain DM specimens; no marked difference was observed. E-cadherin was expressed in the tumor nest, but weakly expressed in the invasion front of the primary tumor. E-cadherin was also expressed in the lung DM specimens, but was not expressed in the brain DM specimens. $\mathrm{N}$-cadherin was not expressed in the primary tumor or lung DM lesions, but it was expressed in the brain DM (Fig. 6B).

\section{Discussion}

Although the NCCN Clinical Practice Guideline has suggested that systemic therapy including cetuximab is the gold standard for very advanced HNSCC (1), there are few studies on patients receiving long-term cetuximab treatment. Here, we determined the histological and immunohistochemical responses in a case of isolated brain DM of OSCC with loco-regional and lung control. The incidence of DM in patients with OSCC after standard therapy has recently been reported to range from 3.2 to $9.6 \%(5-8)$. Among these reports, the incidence of isolated DM has been reported as 3.2\% (7), and the incidence of brain DM has been reported to range from 1.3 to $3.3 \%$ (5,7). From these reports, it can be suggested that our case was extremely unusual.

There are several potential reasons for the occurrence of brain DM in our case. Although tumor cellularity was decreased and certain anti-tumor effects of cetuximab were observed, tumor recurrence was detected only in the brain region during systemic therapy in our case, and the blood brain barrier (BBB) was suspected to be a factor of weakening effect. Regarding cetuximab and the $\mathrm{BBB}$, some authors reported that cetuximab molecules (152 $\mathrm{kDa}$ and $15 \mathrm{~nm})$ are large relative to the size of the BBB junctions, and may not cross the BBB in conventional systemic therapy $(9,10)$. In addition, the BBB in malignant brain tumors [also referred to as the blood-tumor barrier (BTB)] is abnormal and variably disrupted within the main body of the tumor and nearby tissue, and the BTB may be shielded from the effects of chemotherapy (11). For these reasons, the frequency of brain metastasis may be increased in R/M OSCC during cetuximab treatment. According to the NCCN guideline, fluoro-deoxyglucose- positron emission tomography/CT has been recommended to assess the extent of disease or DM at a minimum of 12 weeks if the patient responds to systemic therapy/RT (1). However, it may not be useful in detecting brain metastasis. Although enhanced MRI may be useful for detecting metastasis, brain and loco-regional metastases may not be examined at the same time, depending on institutional imaging conditions. Therefore, the frequency of brain screening will be controversial. In addition, speech disturbances or decreased cognitive function would be apparent when brain metastasis is suspected, and it is important to avoid missing these symptoms at an early stage. 


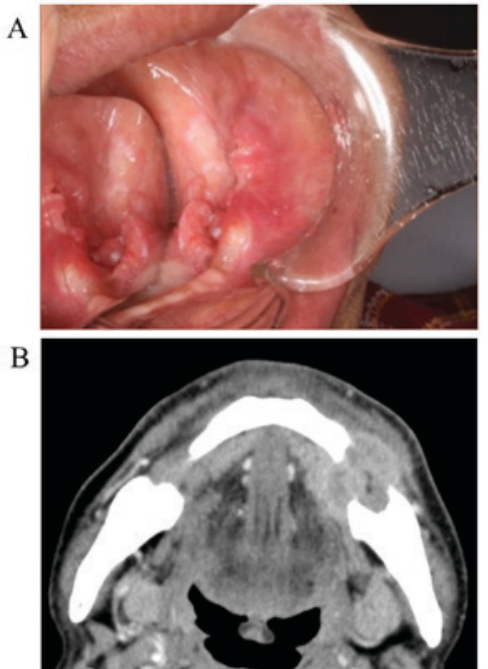

Figure 1. Representative clinical and imaging results. (A) Intraoral examination revealed an elastic hard mass with a central ulceration involving the left mandibular gingiva. (B) Contrast-enhanced axial computed tomography showed a $32 \times 23 \mathrm{~mm}$ poorly marginated lesion with bone destruction that extended along the buccal and lingual surfaces of the mandible.
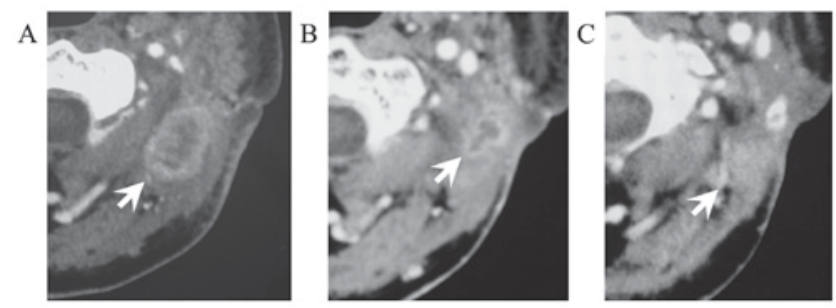

Figure 2. Representative imaging results of regional recurrence lesion (A) Enhanced computed tomography showed a recurrent lesion located between the splenius muscle and levator scapulae muscle 2 months after surgery. The arrow indicates a recurrent lesion located between the splenius muscle and levator scapulae muscle 2 months after surgery. (B) High-dose platinum-based chemoradiotherapy produced marked shrinkage.(C) Residual tumor could not be detected 4 months after cetuximab administration.
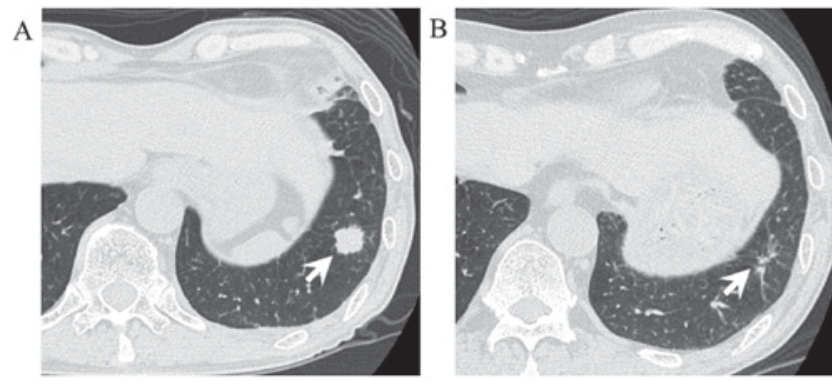

Figure 3. Representative imaging results of lung distant metastases. (A) Axial chest computed tomography detected multiple pulmonary nodules 6 months after concurrent chemoradiotherapy. The arrow indicates multiple pulmonary nodules during the chemoradiotherapy. (B) The nodules could not be detected 3 months after cetuximab administration.

The effects of long-term administration of cetuximab on brain metastasis were immunohistochemically examined. EGFR expression in the metastatic brain lesions in our patient was remarkably decreased compared with the primary tumor and metastatic lung lesions. Benavente et al reported
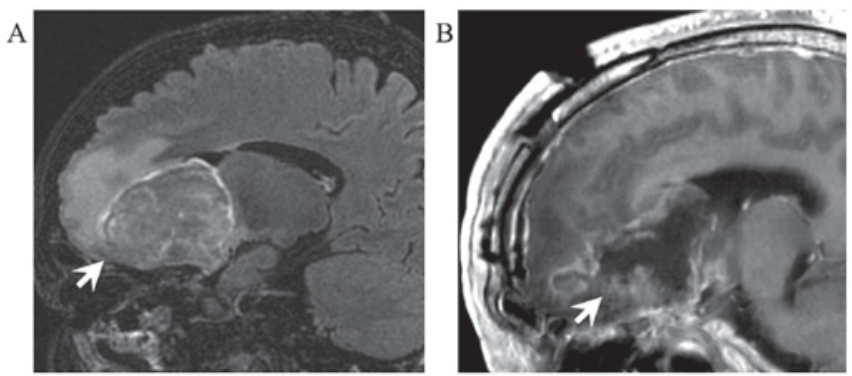

Figure 4. Representative imaging results of brain distant metastases (A) Gadolinium-enhanced T1-weighted magnetic resonance imaging of the head. The arrow indicates a $62 \times 40 \times 40 \mathrm{~mm}$ ring-enhanced mass lesion in the frontal lobe bilaterally. (B) The tumor was subjected to palliative surgery.
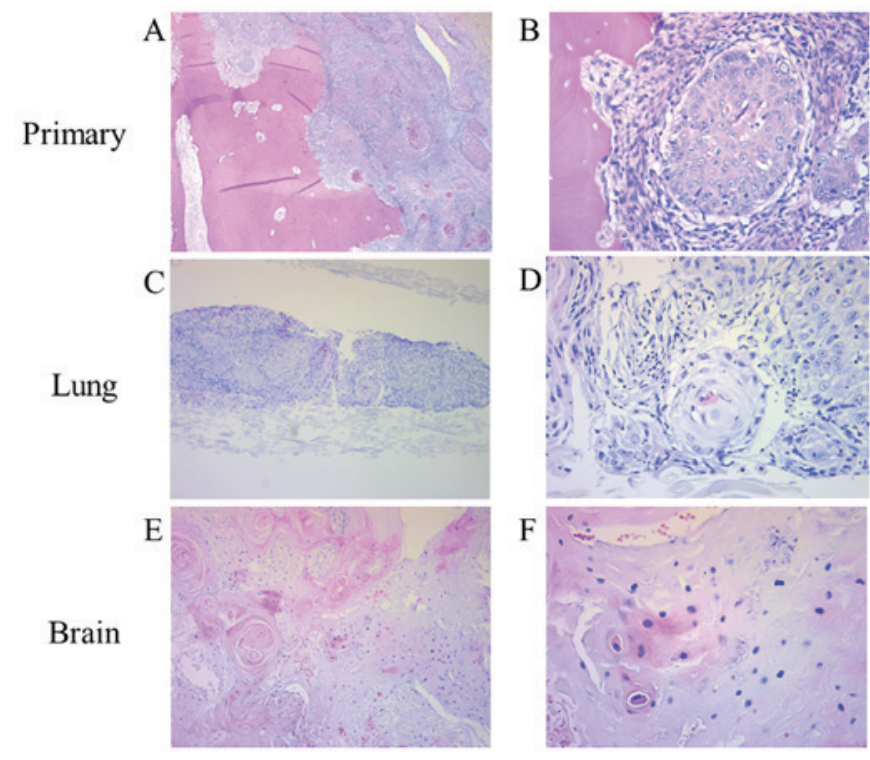

Figure 5. Representative histopathological features of oral squamous cell carcinoma. (A) Hematoxylin and eosin (H\&E) staining (x100) and (B) (x400) for primary tumors. (C) H\&E staining (x100) and (D) (x400) for lung distant metastasis (DM). (E) H\&E staining (x 100) and (F) (x400) (F) for brain DM.

that, when EGF-stimulated activation of EGFR was inhibited, other signaling molecules, such as protein kinase B, mitogen-activated protein kinase, and phosphorylated signal transducer and activator of transcription 3 were upregulated, as in cetuximab-resistant HNSCC cell lines (12). Moreover, Hatakeyama et al reported that EGFR activation leads to activation of downstream pathways, and that downstream effectors are implicated in resistance to EGFR inhibition (13). In contrast, Kimura et al reported that cetuximab-resistant cell lines exhibited reduced levels of EGFR expression and increased epithelial-to-mesenchymal transition (EMT)-associated genes, indicating the association of treatment responses (14). In the present study, we found that metastatic brain lesions were composed of selectively residual EGFR-low-expressing cells refractory to cetuximab. Our results are in agreement with those in previous reports.

With regard to EMT, Schmitz et al also reported a correlation between upregulation of EMT markers and cetuximab resistance using 20 pre- and post-cetuximab HNSCC biopsy specimens (15). However, as the patient underwent the short-term cetuximab treatment before 
A
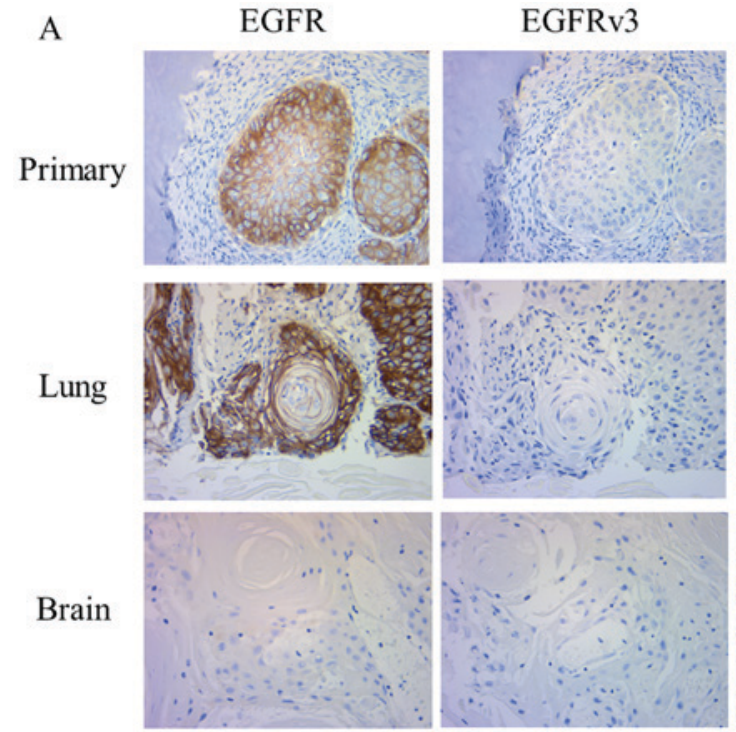

CD44v6
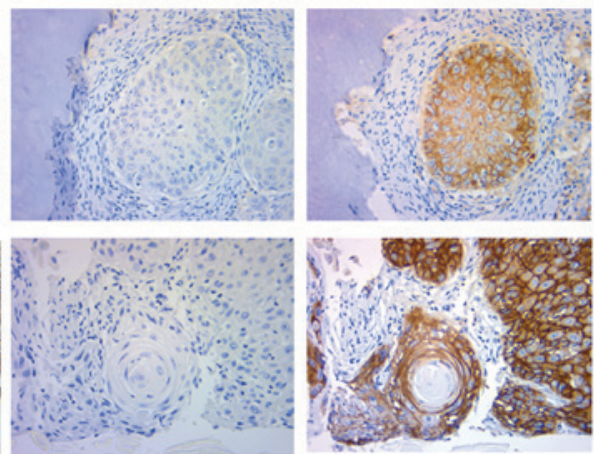

B
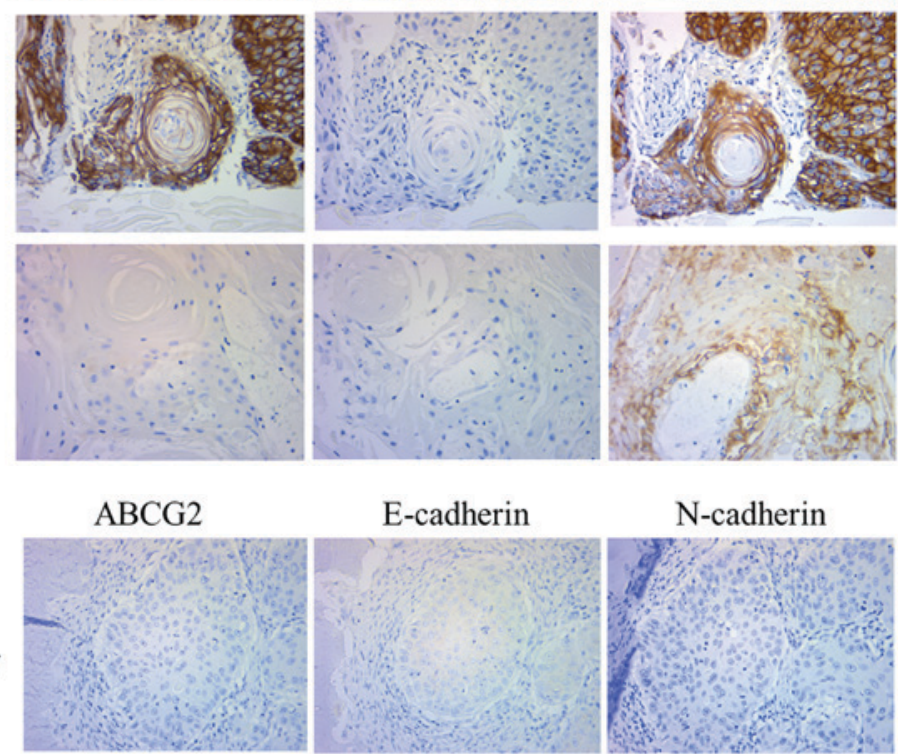

Primary
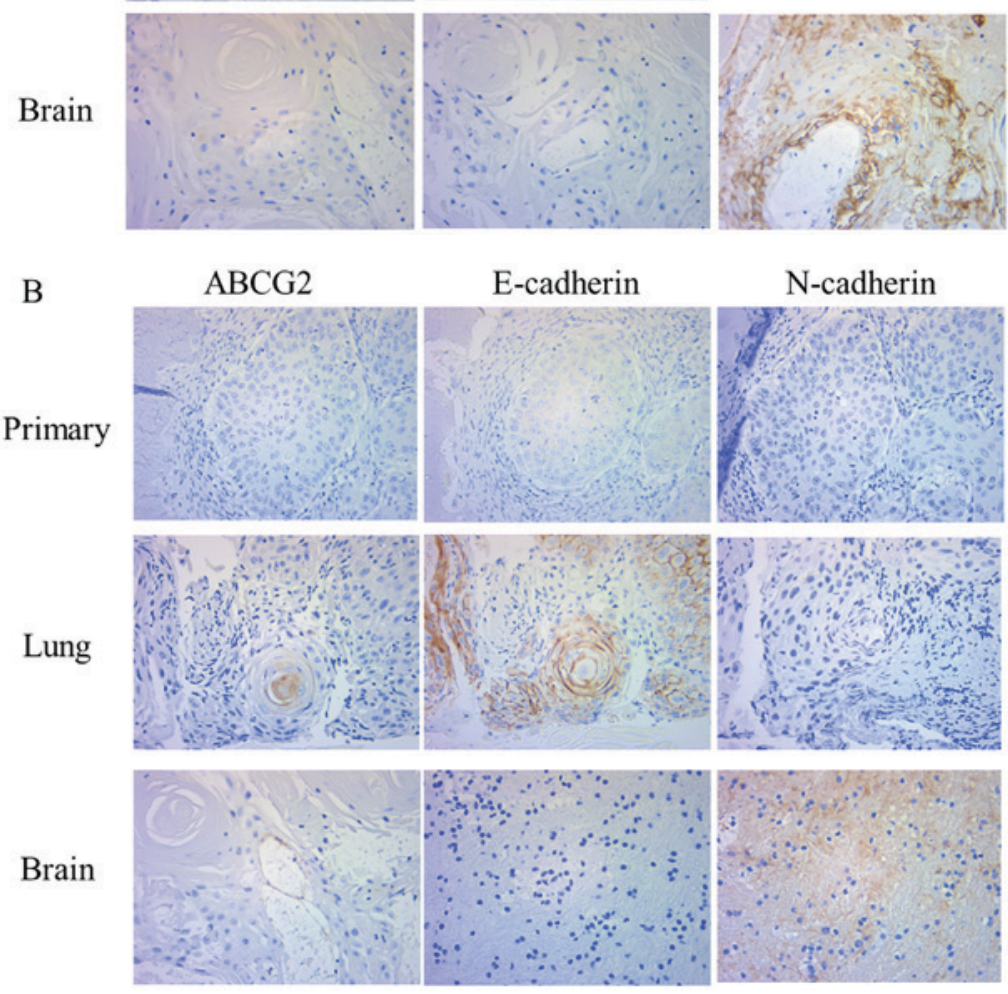

Figure 6. Representative immunohistochemical features of oral squamous cell carcinoma. (A) Epidermal growth factor receptor expression was decreased in the brain distant metastasis (DM) compared to the primary tumor and lung DM (magnification, $\mathrm{x} 400$ ). (B) E-cadherin expression was decreased and $\mathrm{N}$-cadherin expression was increased between lung and brain DM lesion (magnification, $\mathrm{x} 400$ ).

surgery, the relationship between long-term cetuximab treatment and acquired resistance to cetuximab remains unclear. In the present study, E-cadherin was expressed in the lung DM lesions, whereas it was weakly or not expressed in the primary tumor and brain DM lesions. Although it was suspected that primary tumor cells could already have EMT potential, it appears to have been lost in lung DM cells. These results could be attributed to the influence of high-dose CRT. Interestingly, N-cadherin expression in the metastatic brain lesions was increased compared to the primary tumor and metastatic lung lesions, and a cadherin switch profile (low E-cadherin and high $\mathrm{N}$-cadherin expression concurrently), known as a feature of EMT was observed. As it has been reported that cadherin switching from E-cadherin to $\mathrm{N}$-cadherin may play a critical role in development and metastasis of OSCC (16), one of the features of cetuximab resistant cells is suggested.

EGFRv3 is the most common EGFR alteration. This mutation makes the receptor unable to bind its ligand and it therefore signals constitutively. It is co-expressed with wild type EGFR in many cancers, including HNSCC. Wheeler et al reported that activation of the EGFR pathway thorough EGFRv3 led to activation of target genes and subsequent cetuximab resistance $(17,18)$. However, in the present study, there was no difference in EGFRv3 expression between the lung and brain lesions. This result suggested that there is no correlation between acquired resistance to cetuximab and EGFRv3. Moreover, because it was suspected that the occurrence of brain metastasis during systemic therapy was related to cancer stem cells (CSCs), we examined the expressions of CSC markers (CD44v6 and ABCG2) (19). However, we observed no difference in CD44v6 and ABCG2 expression between the lung and brain lesions. However, La Fleur et al reported that CD44high/EGFRlow cells show an EMT phenotype and resistance to cetuximab treatment, and our results are agreement with this finding (20).

In conclusion, the results of this study suggest that acquired resistance to cetuximab is associated with low EGFR 
expression and EMT potential. One limitation of our work is that we examined only one patient, and many biases must exist, including tumor heterogeneity. In addition, because our study examined an R/M OSCC terminal stage patient during long-term cetuximab treatment, the number of tumor sections we could safely collect in a real clinical situation was limited. Therefore, an intergroup study with an increased number of cases is needed.

\section{Conflicts of interest statement}

The authors declare that they have no competing interests.

\section{References}

1. Pfister DG, Spencer S, Brizel DM, Burtness B, Busse PM, Caudell JJ, Cmelak AJ, Colevas AD, Dunphy F, Eisele DW, et al: Head and neck cancers, version 1.2015. J Natl Compr Canc Netw 13: 847-855; quiz 856, 2015.

2. Vermorken J, Mesia R, Rivera F, Remenar E, Kawecki A, Rottey S, Erfan J, Zabolotnyy D, Kienzer HR, Cupissol D, et al: Platinum-based chemotherapy plus cetuximab in head and neck cancer. N Engl J Med 359: 1116-1127, 2008.

3. Hitt R, Irigoyen A, Cortes-Funes H, Grau JJ, García-Sáenz JA and Cruz-Hernandez JJ; Spanish Head and Neck Cancer Cooperative Group (TTCC): Phase II study of the combination of cetuximab and weekly paclitaxel in the first-line treatment of patients with recurrent and/or metastatic squamous cell carcinoma of head and neck. Ann Oncol 23: 1016-1022, 2012.

4. Price KA and Cohen EE: Mechanisms of and therapeutic approaches for overcoming resistance to epidermal growth factor receptor (EGFR)-targeted therapy in squamous cell carcinoma of the head and neck (SCCHN). Oral Oncol 51: 399-408, 2015.

5. Hasegawa T, Tanakura M, Takeda D, Sakakibara A, Akashi M, Minamikawa $\mathrm{T}$ and Komori T: Risk factors associated with distant metastasis in patients with oral squamous cell carcinoma. Otolaryngol Head Neck Surg 152: 1053-1060, 2015.

6. Liao CT, Wang HM, Chang JT, Ng SH, Hsueh C, Lee LY, Lin CH, Chen IH, Huang SF and Yen TC: Analysis of risk factors for distant metastases in squamous cell carcinoma of the oral cavity. Cancer 110: 1501-1508, 2007.

7. Lim JY, Lim YC, Kim SH, Kim JW, Jeong HM and Choi EC: Predictive factors of isolated distant metastasis after primary definitive surgery without systemic treatment for head and neck squamous cell carcinoma. Oral Oncol 46: 504-508, 2010.

8. Sakamoto Y, Matsushita Y, Yamada SI, Yanamoto S, Shiraishi T, Asahina I and Umeda M: Risk factors of distant metastasis in patients with squamous cell carcinoma of the oral cavity. Oral Surg Oral Med Oral Pathol Oral Radiol 121: 474-480, 2016.

9. Chakraborty S, Filippi CG, Wong T, Ray A, Fralin S, Tsiouris AJ, Praminick B, Demopoulos A, McCrea HJ, Bodhinayake I, et al: Superselective intraarterial cerebral infusion of cetuximab after osmotic blood/brain barrier disruption for recurrent malignant glioma: Phase I study. J Neurooncol 128: 405-415, 2016.
10. Grisanti S, Amoroso V, Buglione M, Rosati A, Gatta R, Pizzocaro C, Ferrari VD and Marini G: Cetuximab in the treatment of metastatic mucoepidermoid carcinoma of the salivary glands: A case report and review of literature. J Med Case Rep 2: 320, 2008.

11. Black KL and Ningaraj NS: Modulation of brain tumor capillaries for enhanced drug delivery selectively to brain tumor. Cancer Control 11: 165-173, 2004.

12. Benavente S, Huang S, Armstrong EA, Chi A, Hsu KT, Wheeler DL and Harari PM: Establishment and characterization of a model of acquired resistance to epidermal growth factor receptor targeting agents in human cancer cells. Clin Cancer Res 15: 1585-1592, 2009.

13. Hatakeyama H, Cheng H, Wirth P, Counsell A, Marcrom SR, Wood CB,Pohlmann PR, Gilbert J, Murphy B, Yarbrough WG, et al: Regulation of heparin-binding EGF-like growth factor by miR-212 and acquired cetuximab-resistance in head and neck squamous cell carcinoma. PLoS One 5: e12702, 2010.

14. Kimura I, Kitahara H, Ooi K, Kato K, Noguchi N, Yoshizawa K, Nakamura $\mathrm{H}$ and Kawashiri S: Loss of epidermal growth factor receptor expression in oral squamous cell carcinoma is associated with invasiveness and epithelial-mesenchymal transition. Oncol Lett 11: 201-207, 2016.

15. Schmitz S, Bindea G, Albu RI, Mlecnik B and Machiels JP: Cetuximab promotes epithelial to mesenchymal transition and cancer associated fibroblasts in patients with head and neck cancer. Oncotarget 6: 34288-34299, 2015.

16. Pyo SW, Hashimoto M, Kim YS, Kim CH, Lee SH, Johnson KR, Wheelock MJ and Park JU: Expression of E-cadherin, P-cadherin and N-cadherin in oral squamous cell carcinoma: Correlation with the clinicopathologic features and patient outcome. J Craniomaxillofac Surg 35: 1-9, 2007.

17. Wheeler SE, Suzuki S, Thomas SM, Sen M, Leeman-Neill RJ, Chiosea SI, Kuan CT, Bigner DD, Gooding WE, Lai SY and Grandis JR: Epidermal growth factor receptor variant III mediates head and neck cancer cell invasion via STAT3 activation. Oncogene 29: 5135-5145, 2010.

18. Wheeler SE, Morariu EM, Bednash JS, Otte CG, Seethala RR, Chiosea SI and Grandis JR: Lyn kinase mediates cell motility and tumor growth in EGFRvIII-expressing head and neck cancer. Clin Cancer Res 18: 2850-2860, 2012.

19. Yanamoto S, Yamada S, Takahashi H, Naruse T, Matsushita Y, Ikeda H, Shiraishi T, Seki S, Fujita S, Ikeda T, et al: Expression of the cancer stem cell markers CD44v6 and ABCG2 in tongue cancer: Effect of neoadjuvant chemotherapy on local recurrence. Int J Oncol 44: 1153-1162, 2014.

20. La Fleur L, Johansson AC and Roberg K: A CD44high/EGFRlow subpopulation within head and neck cancer cell lines shows an epithelial-mesenchymal transition phenotype and resistance to treatment. PLoS One 7: e44071, 2012.

(i) (2) This work is licensed under a Creative Commons Attribution-NonCommercial-NoDerivatives 4.0 International (CC BY-NC-ND 4.0) License. 